patients demonstrated significantly $(p<0.05)$ greater baseline disease severity with respect to mean (SD) joint count [TJC: 8.9 (6.2) vs. 7.4 (6.6); SJC: 7.4 (5.0) vs. 5.9 (4.6)], morning stiffness [83.5 (98.2) vs. 61.8 (77.4) min/day], PtGA [56.1 (24.1) vs. 45.1 (24.7) mm], pain [58.5 (24.3) vs. 50.1 (24.0) mm], DLQI scores [6.1 (6.9) vs. $4.3(5.3)]$ and HAQ-DI $[1.1(0.6)$ vs. $0.8(0.6)]$. The rate of baseline mMDA was slightly lower for ADA patients (4.3\% vs. $7.4 \% ; p=0.178)$. Baseline prevalence of enthesitis was comparable (ADA: $28.4 \%$ vs. csDMARD: $23.4 \%$; $\mathrm{p}=0.276$ ), while dactylitis was significantly more prevalent for csDMARD patients (26.2\% vs. $36.3 \%$; $p=0.031$ )

Overall effect of treatment group, over 24 months, significantly $(p<0.05)$ favored the ADA vs. csDMARD-treated patients for TJC [estimate $(95 \% \mathrm{Cl}):-2.4(-3.4$, -1.4)] SJC [-1.8 (-2.5, -1.2)], PtGA [-3.7 $(-9.3,1.9)]$, DLQI [-1.5 (-2.5, -0.5)], and $\mathrm{HAQ}-\mathrm{DI}[-0.1(-0.2,0.0)]$ (Figure 1). There was no significant difference for morning stiffness and pain.

At month 24 , statistically comparable $(p>0.05)$ baseline-adjusted values (the least square means: LSM) were observed for ADA- vs. csDMARD-treated patients for TJC [LSM $(95 \% \mathrm{Cl}): 1.8(1.2,2.4)$ vs. $3.0(2.1,3.8)]$, SJC $[1.2(0.8,1.7)$ vs. $2.1(1.5,2.7)]$, morning stiffness $[32.4(19.1,45.6)$ vs. $29.9(11.1,48.6) \mathrm{min} /$ day], PtGA [31.6 $(28.1,35.2)$ vs. $36.9(31.8,41.9) \mathrm{mm}$ ], pain [35.3 $(31.5,39.0)$ vs. $38.4(33.1,43.7) \mathrm{mm}$ ], DLQI [2.9 $(2.2,3.6)$ vs. $2.9(2.0,3.8)]$, and HAQ-DI [0.7 $(0.6,0.8)$ vs. $0.9(0.8,1.0)]$.

Achievement of mMDA at month 24 was reported by $34.1 \%$ and $34.9 \%$ of ADA- and csDMARD-treated patients, respectively $(\mathrm{p}=0.892)$. Rates of dactylitis $(10.6 \%$ vs. $10.0 \%)$ and enthesitis $(9.6 \%$ vs. $14.4 \%)$ were comparable in the ADA vs. csDMARDs groups respectively.

Conclusion: The results of this real-world Canadian study indicate a physician selection bias for treatment with ADA for PsA patients with more severe disease burden, indicated by greater baseline disease activity and PROs. ADA-treated patients experienced a greater treatment effect over 24 months compared to csDMARD-treated patients. However, despite the greater treatment effect of ADA, residual disease burden in the two groups was comparable at 24 months.

Acknowledgements: The authors wish to acknowledge JSS Medical Research for their contribution to the statistical analysis, medical writing, and editorial support during the preparation of this abstract. AbbVie provided funding to JSS Medical Research for this work.

Disclosure of Interests: Majed Khraishi Speakers bureau: Speaker for AbbVie, Consultant of: Consultant for AbbVie, Grant/research support from: Principal Investigator for AbbVie, Samuel Silverberg Consultant of: Consultant for AbbVie, Janssen, and Pfizer, Yatish Setty Consultant of: Advisory Board meetings with AbbVie and Janssen, Marie-Claude Laliberté Employee of: Employee of AbbVie, Louis Bessette Speakers bureau: Speaker for Amgen, BMS, Janssen, UCB, AbbVie, Pfizer, Merck, Celgene, Lilly, Novartis, Gilead, Sandoz, Fresenius Kabi, Consultant of: Consultant for Amgen, BMS, Janssen, UCB, AbbVie, Pfizer, Celgene, Lilly, Novartis, Gilead, Sandoz, Samsung Bioepis, Fresenius Kabi, Grant/research support from: Investigator for Amgen, BMS, Janssen, UCB, AbbVie, Pfizer, Merck, Celgene, Sanofi, Lilly, Novartis, Gilead.

DOI: 10.1136/annrheumdis-2021-eular.2589

\section{AB0556 COMPARING EFFICACY OF GUSELKUMAB VERSUS USTEKINUMAB IN PATIENTS WITH PSORIASIS ARTHRITIS: AN ADJUSTED COMPARISON USING INDIVIDUAL PATIENT DATA FROM DISCOVER 1\&2 AND PSUMMIT TRIALS}

J. Diels ${ }^{1}$, P. Thilakarathne ${ }^{2}$, A. Schubert ${ }^{3}$, F. Hassan $^{4}$, S. Peterson ${ }^{5}$, W. Noel'. ' Janssen Pharmaceutica, HEMAR Department, Beerse, Belgium; 2 JanssenCilag Ltd, HEMAR Department, Beerse, Belgium; ' Janssen-Cilag Poland, HEMAR Department, Warsaw, Poland; ${ }^{4}$ Janssen-Cilag Ltd, HEMAR Department, High Wycombe, United Kingdom; ${ }^{5}$ Janssen Global Services, LLC, Janssen Immunology Global Commercial Strategy Organization, Raritan, NJ, United States of America

Background: Guselkumab is an anti-interleukin (IL)-23 monoclonal antibody recently approved for a treatment of Psoriasis arthritis (PsA). In two large Phase III trials of patients with PSA (DISCOVER -1 \& -2) guselkumab has shown to be superior versus placebo. In this indication no direct comparison is available between guselkumab and ustekinumab, a monoclonal antibody targeting IL-12 and IL-23. Indirect comparisons based on relative treatment effects versus a common comparator (placebo) only allow for analyses up to week 24 due to cross-over to active arms in available PsA trials.

Objectives: To compare indirectly joint and skin efficacy of guselkumab versus ustekinumab up to week 52, using pooled patient-level trial data from DISCOVER $1 \& 2$ and PSUMMIT 1\&2, adjusting for cross-trial population differences.
Methods: Patient level data, including baseline characteristics and outcome data on American College of Rheumatology (ACR) response, Psoriasis Area Severity Index (PASI) response from the guselkumab arms of DISCOVER \& -2 were pooled with the data from the ustekinumab trials PSUMMIT -1\& 2. Analyses were performed for bio-naïve and bio-experienced populations separately. Differences in patient characteristics across trial populations were adjusted for using multivariate logistic regression, including: gender, age body mass index, previous TNF use, disease duration, PASI level, number of swollen and tender joints. This method of indirect comparisons allows for analysis of comparative efficacy beyond controlled induction period and odds ratios' resulting from this model were translated into predicted response rates for ustekinumab, assuming same patient population, as enrolled in the guselkumab trial arms.

Results: Majority of baseline characteristics for patients on guselkumab (100mg q8w; 100mg q4w) were comparable to patients on ustekinumab $45 / 90 \mathrm{mg}$, in both in bio-naïve and bio-experienced group of patients. The probability of reaching a ACR 20 in both the bio-naïve \& bio-experienced population was significantly higher for guselkumab vs ustekinumab at weeks 52 for both dosing regimens of guselkumab (bio-naïve ACR 20: q8w OR=1.88 [1.28;2.76]) $q 4 \mathrm{w}(\mathrm{OR}=1.92$ [1.29;2.86]; bio experienced ACR20 q8w OR=2.72[1.17;6.31] $q 4 \mathrm{w}$ OR=4.77 [1.95;11.63]). Similarly guselkumab was superior over ustekinumab on PASI 90 outcome at week 52 in both bio-naïve \& bio-experienced patients with $B S A \geq 3 \%$ at baseline (bio-naïve: q8w OR=2.59 [1.68;3.99]), q4w $\mathrm{OR}=3.19$ [2.03;5.00], and bio-experienced $q 8 \mathrm{w}$ OR=3.96[1.39,11.27], q4w OR=13.10[4.18,41.04]). Figure 1 represents unadjusted pooled DISCOVER $1 \& 2$ trial results and estimated proportions of ustekinumab treated patient group achieving ACR 20 in bio-naïve patient group up to week 52 using the method described above.

Conclusion: An adjusted comparison using patient level data from pivotal Phase III studies demonstrates both dosages of guselkumab to be significantly more effective versus ustekinumab in both skin and joint outcomes in both bio-naïve \& bio experienced patients up to week 52

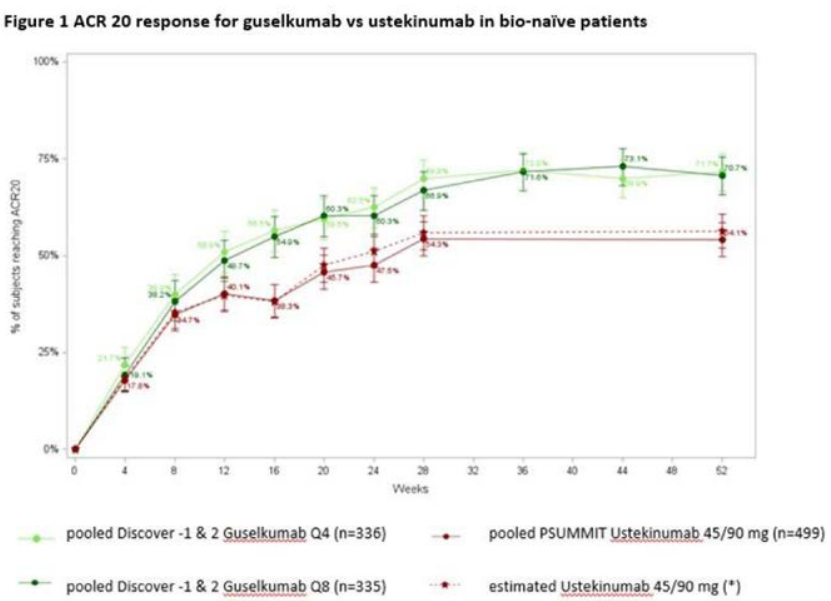

Disclosure of Interests: Joris Diels Shareholder of: Janssen, Employee of: Janssen, Pushpike Thilakarathne Employee of: Janssen, Agata Schubert Shareholder of: Janssen, Employee of: Janssen, Fareen Hassan Shareholder of: Janssen, Employee of: Janssen, Steve Peterson Shareholder of: Janssen, Employee of: Janssen, Wim Noel Shareholder of: Janssen, Employee of: Janssen.

DOI: 10.1136/annrheumdis-2021-eular.2595

\section{$\mathrm{AB} 0557$ \\ ACHIEVING TREATMENT TARGETS IN PSORIATIC ARTHRITIS WITH APREMILAST IN CANADIAN PRACTICE: REAL WORLD RESULTS FROM APPRAISE}

V. Chandran ${ }^{1}$, L. Bessette ${ }^{2}$, C. Thorne ${ }^{3}$, M. Sheriff ${ }^{4}$, P. Rahman ${ }^{5}$, D.

D. Gladman ${ }^{1}$, S. Anwar ${ }^{6}$, J. Jelley ${ }^{7}$, A. J. Gaudreau ${ }^{7}$, M. Chohan ${ }^{7}$, J.

S. Sampalis ${ }^{8} .{ }^{1}$ Schroeder Arthritis Institute, Rheumatology, Toronto, Canada;

${ }^{2}$ Laval University, Rheumatology, Quebec City, Canada; ${ }^{3}$ Southlake Regional Health Centre, Rheumatology, Newmarket, Canada; ${ }^{4}$ Nanaimo Regional General Hospital, Rheumatology, Nanaimo, Canada; ${ }^{5}$ Memorial University of Newfoundland, Rheumatology, New Foundland, Canada; ${ }^{6}$ Windsor Regional Hospital, Rheumatology, Windsor, Canada; ${ }^{7}$ Amgen Canada Inc., Global Medical Affairs, Mississauga, Canada; ${ }^{8}$ McGill University and JSS Medical Research, Epidemiology, Montreal, Canada 
Background: Real-world evidence on achieving treatment targets with apremilast (APR) in patients (pts) with PsA is limited. In the phase 3 PALACE trials, pts reached remission (REM)/low disease activity (LDA) targets at 52 wks most frequently when early APR treatment was initiated and pts were in moderate disease activity, as measured by Clinical Disease Activity Index for PsA (cDAPSA) score. In APPRAISE, we assessed APR effectiveness/tolerability in pts with PsA in routine clinical practice in Canada.

Objectives: This interim efficacy analysis focused on the available data on APR effectiveness measuring rate of achieving cDAPSA REM or LDA at 12 mos and Pt Acceptable Symptom Status (PASS) results.

Methods: The prospective, multicenter, observational APPRAISE study assessed APR effectiveness/tolerability in adults with active PsA in routine clinical care enrolled from July 2018-March 2020. Pts were followed from treatment initiation to 12 mos, with visits suggested every 4 mos. The primary effectiveness endpoint was the rate of achieving at least LDA (cDAPSA <14) at 12 mos. Pt-reported outcome measures were assessed. Data reported are as observed in pts continuing APR treatment.

Results: In total, 101 pts were enrolled in APPRAISE. Mean age was 52 yrs; $56 \%$ were women. Mean (SD) PsA duration at baseline (BL) was 6 (8) yrs. Oligoarticular disease ( $\leq 4$ joint involvement) was most common (41\%), followed by polyarticular (35\%). Most pts (92\%) received prior conventional DMARDs and 17\% received prior biologic therapy; concomitant MTX was reported in $41 \%$ at BL. By 12 mos, 41/101 enrolled pts discontinued, 35 reached 12 mos follow-up (4 mos: $n=92 ; 8$ mos: $n=61)$, and 25 have yet to reach 12 mos. The majority $(92 \%)$ of discontinuations due to lack/loss of effectiveness or AEs occurred within 4-8 mos. AEs were primarily Gl related early in treatment. The proportion of pts with continued APR achieving CDAPSA REM/LDA treatment targets increased significantly over time (Figure 1). Significant reductions were seen over 12 mos in swollen/tender joint counts and plaque psoriasis, with reduced mean (SD) body surface area of $-4 \%$ (9\%) (Table 1). Prevalence of dactylitis/enthesitis at BL, 4, 8, and 12 mos was $17 \% / 33 \%, 9 \% / 24 \%, 5 \% / 19 \%$, and $0 \% / 21 \%$, respectively. Pain assessment (VAS) significantly improved over time. The proportion of pts achieving PASS with continued APR increased significantly over 12 mos (BL: 27\%; 12 mos: 65\%) (Figure 1). COVID restrictions impacted in-office assessment visits, necessitating reliance on virtual visits.

Conclusion: Pts with PsA receiving APR were assessed at regular intervals in routine clinical care in Canada. This interim analysis revealed a greater number of pts receiving APR (66\%) who completed the 12-mo follow-up achieved REM or LDA, as measured by cDAPSA over 12 mos. A majority of pts $(65 \%)$ reported satisfaction with their disease state, as measured by PASS. No new safety signals were observed.

Table 1. Change in Clinical Parameters and Pt-Reported Outcomes From BL to 4,8 , and 12 Mos

Outcome Measure ${ }^{\star}$ Mean (SD)BL $(n=101) 4 \operatorname{Mos}(n=92) 8 \operatorname{Mos}(n=61) 12 \operatorname{Mos}(n=35)$

\begin{tabular}{lllll}
\hline $\begin{array}{l}\text { Disease/Clinical Parameters } \\
\text { Tender joint count (0-68) }\end{array}$ & $7.5(6.7)$ & $-2.5(6.3)^{\star}$ & $-3.9(5.2)^{\star}$ & $-2.2(6.4)$ \\
Swollen joint count (0-66) & $5.4(5.4)$ & $-3.0(4.5)^{\star}$ & $-3.1(4.3)^{\star}$ & $-3.1(4.4)^{\star}$ \\
PhGA & $42.9(18.8)$ & $-19.0(24.6)^{\star}$ & $-24.2(24.2)^{\star}$ & $-21.2(26.3)^{\star}$ \\
$\quad \begin{array}{llll}\text { Body surface area, \% } \\
\quad \text { cDAPSA }\end{array}$ & $3.1(6.1)$ & $-2.2(6.0)^{\star}$ & $-2.7(7.5)^{\star}$ & $-4.2(9.1)^{\star}$ \\
Pt-Reported Outcomes & $22.2(13.3)$ & $-7.9(12.1)^{\star}$ & $-10.1(13.5)^{\star}$ & $-6.9(12.0)^{\star}$ \\
PtGA, mm & & & & \\
Pain, mm & $50.0(24.6)$ & $-10.2(27.5)^{\star}$ & $-9.1(31.9)^{\star}$ & $-3.6(39.7)$ \\
HAQ-DI & $48.3(25.3)$ & $-9.5(26.2)^{\star}$ & $-12.2(28.7)^{\star}$ & $-7.3(26.0)$ \\
& $0.9(0.7)$ & $-0.13(0.5)^{\star}$ & $-0.15(0.6)$ & $-0.1(0.7)$
\end{tabular}

${ }^{*}$ Denotes significant change from BL $(P<0.05)$ from paired-sample $t$-tests; note that mean change from $B L$ may be greater than the mean $B L$ value when improvements of large magnitude, for pts with relatively elevated BL values, are observed in samples with lower n's. HAQ-DI = Health Assessment Questionnaire-Disability Index; PhGA = Physician's Global Assessment; PtGA = Patient's Global Assessment.

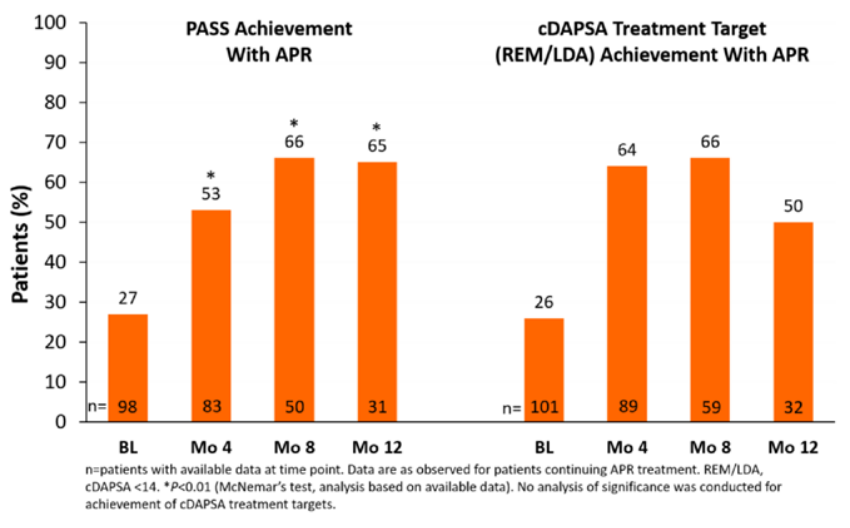

Acknowledgements: This study was funded by Celgene and Amgen Inc. Writing support was funded by Amgen Inc. and provided by Kristin Carlin, RPh, MBA, of Peloton Advantage, LLC, an OPEN Health company.

Disclosure of Interests: Vinod Chandran Consultant of: AbbVie, Amgen, BMS, Celgene Corporation, Eli Lilly, Janssen, Novartis, Pfizer, UCB, Grant/ research support from: AbbVie, Amgen, BMS, Celgene Corporation, Eli Lilly, Janssen, Novartis, Pfizer, UCB, Louis Bessette Consultant of: AbbVie, Amgen, BMS, Celgene Corporation, Eli Lilly, Janssen, Merck, Pfizer, Sanofi, Novartis, UCB, Grant/research support from: AbbVie, Amgen, BMS, Celgene Corporation, Eli Lilly, Janssen, Merck, Pfizer, Sanofi, Novartis, UCB, Carter Thorne Speakers bureau: AbbVie, Amgen, Celgene Corporation, Eli Lilly, Medexus/ Medac, Merck, Novartis, Pfizer, Sandoz, Sanofi, Consultant of: Centocor Medexus/Medac, Merck, Grant/research support from: Novartis, Pfizer, Maqbool Sheriff Speakers bureau: AbbVie, Celgene Corporation, Eli Lilly, Janssen Merck, Pfizer, Sandoz, Proton Rahman Speakers bureau: AbbVie, Amgen, Celgene, Eli Lilly, Janssen, Merck, Novartis, Pfizer, UCB, Dafna D Gladman Consultant of: AbbVie, Amgen, BMS, Celgene Corporation, Eli Lilly, Galapagos, Gilead, Janssen, Novartis, Pfizer, UCB, Grant/research support from: AbbVie, Amgen, BMS, Celgene Corporation, Eli Lilly, Galapagos, Gilead, Janssen, Novartis, Pfizer, UCB, Sabeen Anwar Consultant of: AbbVie, Amgen Inc., BMS, Novartis, and Pfizer, Grant/research support from: AbbVie, Amgen Inc., Eli Lilly, Janssen, and Pfizer, Jennifer Jelley Employee of: Amgen Canada Inc., Anne-Julie Gaudreau Employee of: Amgen Canada Inc., Manprit Chohan Employee of: Amgen Canada Inc., John S. Sampalis Employee of: JSS Medical Research.

DOI: 10.1136/annrheumdis-2021-eular.2599

\section{AB0558 \\ CLINICAL EFFECTIVENESS AND SAFETY OF ADALIMUMAB VERSUS NON-BIOLOGIC THERAPY IN PSORIATIC ARTHRITIS PATIENTS OVER 24 MONTHS - RESULTS OF THE COMPLETE-PSA CANADIAN OBSERVATIONAL STUDY}

M. Khraishi ${ }^{1}$, J. Stewart ${ }^{2}$, Y. Setty ${ }^{3}$, M. C. Laliberté ${ }^{4}$, L. Bessette ${ }^{5} .{ }^{1}$ Memorial University of Newfoundland, Memorial University of Newfoundland, St. John's, Canada; ${ }^{2}$ University of British Columbia, University of British Columbia, Penticton, Canada; ${ }^{3}$ Grey Bruce Health Services, Grey Bruce Health Services, Owen Sound, Canada; ${ }^{4}$ AbbVie Corporation, AbbVie Corporation, Montreal, Canada; ${ }^{5}$ Laval University, Centre Hospitalier de l'Université Laval, Laval University, Centre Hospitalier de I'Université Laval, Quebec City, Canada

Background: COMPLETE-PSA was an observational study of biologic-naïve Canadian adults with active psoriatic arthritis (PSA) treated with adalimumab or non-biologic disease-modifying anti-rheumatic drugs (nbDMARD) after switch from initial therapy.

Objectives: The aim of this analysis was to assess the 24-month clinical effectiveness and safety of adalimumab vs. nbDMARD in the treatment of PsA in a real-life setting.

Methods: Eligible patients were biologic naïve adults, with active PsA who required change in their treatment regimen due to inadequate response or intolerance, per the judgment of the treating physician. Patients were enrolled between July/2011 and December/2017 and followed for up to 24 months. Patients were treated as per routine care. The primary endpoint, change in DAS-28 to month 24 , was assessed with baseline-adjusted multivariable models and the least square mean (LSM) estimates were generated; Physician's Global Assessment of disease activity (PGA, 100mm VAS) was assessed using similar methodology. Probability of achieving the following therapeutic response thresholds was ascertained and odds ratios (ORs) were generated: $50 \% / 70 \%$ improvement in the American College of Rheumatology criteria (ACR50/ACR70), DAS-28<3.2 (low disease activity or remission; LDA/ remission), DAS-28<2.6 (remission), modified minimal disease activity (mMDA: achievement of $5 / 7$ of: TJC and SJC $\leq 1$ each, BSA $\leq 3 \%$, pain $\leq 15$ (VAS, mm), Patient Global Assessment [PtGA] $\leq 20, \mathrm{HAQ}-\mathrm{DI} \leq 0.5$, and no enthesitis), and psoriasis (PsO) BSA $<3 \%$.

Results: A total of 277 adalimumab and 148 nbDMARD- treated patients were included as part of the intent-to-treat population. Baseline methotrexate was reported by $61.7 \%$ and $81.1 \%$ of adalimumab and nbDMARD-treated patients, respectively. PsO BSA at baseline was predominantly $<3 \%$ for both adalimumab (60.2\%) and nbDMARD (64.6\%) patients. Adalimumab-treated patients reported significantly $(p<0.05)$ higher mean $(S D)$ disease activity for both DAS-28 [4.8 (1.2) vs. 4.3 (1.1)] and PGA [59.4 (19.5) vs. $51.0(21.8) \mathrm{mm}]$ at baseline.

For the primary endpoint, baseline-adjusted month 24 DAS-28 levels were significantly lower for adalimumab vs. nbDMARD patients [LSM $(95 \% \mathrm{Cl}): 2.4(2.2-2.6)$ vs. 3.0 (2.7-3.3); $p=0.037$ ]. In addition, rapid and sustained reductions in DAS-28 were observed for adalimumab-treatment patients, with overall treatment effect significant $(p<0.001)$ in favor of adalimumab [estimate $(95 \% \mathrm{Cl}):-1.1(-1.4,-0.7)$ ] similar results were observed for PGA $[-12.9(-1.7,-8.0)]$. Adalimumab-treated 